\title{
Hard-Wired Dopant Networks and the Prediction of High Transition Temperatures in Ceramic Superconductors
}

\author{
J. C. Phillips \\ Department of Physics and Astronomy, Rutgers University, Piscataway, NJ 08854-8019, USA \\ Correspondence should be addressed to J. C. Phillips, jcphillips8@comcast.net
}

Received 22 May 2009; Accepted 17 August 2009

Academic Editor: Sasha Alexandrov

Copyright () 2010 J. C. Phillips. This is an open access article distributed under the Creative Commons Attribution License, which permits unrestricted use, distribution, and reproduction in any medium, provided the original work is properly cited.

I review the multiple successes of the discrete hard-wired dopant network model ZZIP, and comment on the equally numerous failures of continuum models, in describing and predicting the properties of ceramic superconductors. The prediction of transition temperatures can be regarded in several ways, either as an exacting test of theory, or as a tool for identifying theoretical rules for defining new homology models. Popular "first principle" methods for predicting transition temperatures in conventional crystalline superconductors have failed for cuprate HTSC, as have parameterized models based on $\mathrm{CuO}_{2}$ planes (with or without apical oxygen). Following a path suggested by Bayesian probability, it was found that the glassy, self-organized dopant network percolative model is so successful that it defines a new homology class appropriate to ceramic superconductors. The reasons for this success in an exponentially complex (non-polynomial complete, NPC) problem are discussed, and a critical comparison is made with previous polynomial (PC) theories. The predictions are successful for the superfamily of all ceramics, including new non-cuprates based on FeAs in place of $\mathrm{CuO}_{2}$.

\section{Introduction}

The prediction of transition temperatures $T_{c}$ is rightly considered to be one of the most difficult problems in theoretical physics. Here one should distinguish between a true (or bare) prediction (the value of $T_{c}$ is not known when the prediction is made), and a postdated calculation carried out according to certain rules after $T_{c}$ has been measured experimentally. If the rules (sometimes called "first principles") have been established for similar materials, and they are faithfully applied to the new case, then the validity of the rules can be tested by the success of the prediction. If the number of example materials where the rules have been applied previously is large compared to the number of adjustable parameters, then the prediction can be said to be based on homology, namely, the supposed microscopic similarity of the phase transition of the new material to those previously studied.

Perhaps the best-known example of a "bare" prediction of a transition temperature was [1] for superfluidity of ${ }^{3} \mathrm{He}$. The predicted value of $T_{c}$ was $100 \mathrm{mK}$, whereas the measured value [2] of $T_{c}$ was $3.6 \mathrm{mK}$, so the predicted value was too large by a factor of $\sim 30$. The most successful predated prediction of $T_{c}$ for superconductors based on homology was for the high-pressure phase of $\mathrm{Si}$ [3], where the experimental maximum $T_{c}$ is $8.2 \mathrm{~K}$, and estimation of $T_{c}$ using the most popular rules gave $T_{c}=5 \mathrm{~K}$. A number of postdated predictions of superconductive $T_{c}$ have been made based on homology models and "first principles" rules. The rules depend mainly on the Fermisurface average of the electron-phonon coupling constant $\lambda$ and the phonon frequency squared $\left(\lambda\left\langle\omega^{2}\right\rangle\right)$. The most popular of these have been the prediction of $T_{c}$ in $\mathrm{MgB}_{2}$ based on homologies with simple metals such as $\mathrm{Al}$, where the predicted and experimental values are both about $40 \mathrm{~K}$ [4]. Equally impressive has been the prediction of $T_{c}$ in $5 \% \mathrm{~B}-$ doped CVD diamond, where the predicted and experimental values are $T_{c}=22 \mathrm{~K}$ and $T_{c}=11 \mathrm{~K}$, respectively [5]. The agreement here between theory and experiment is better than it appears to be, as some of the $B$ may have formed electrically inactive dimers. This example illustrates the strengths and limitations of homology arguments based on first principles, as the material properties of $B$-doped CVD 
Continuum versus network phase diagrams
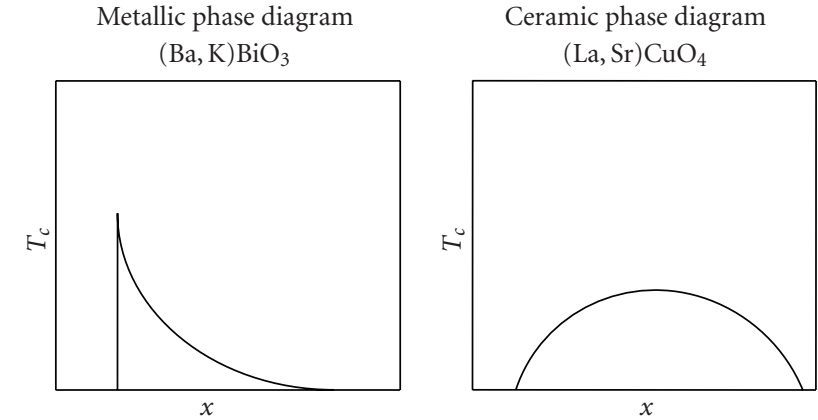

FIGURE 1: There are large qualitative differences between the phase diagrams of layered ceramic cuprates, such as $\mathrm{La}_{2-x} \mathrm{Sr}_{x} \mathrm{CuO}_{4}$, and cubic $\mathrm{Ba}_{1-x} \mathrm{~K}_{x} \mathrm{BiO}_{3}$. The former requires a percolative model, but the superconductive properties of the latter can be explained using a traditional BCS continuum model.

diamond are a far cry from those of single-crystal $\mathrm{Al}$ or $\mathrm{MgB}_{2}$.

The success of crystalline models for electron-phonon interactions in $\mathrm{MgB}_{2}$, and their failure in ceramic superconductors, is sometimes cited as "proof" that ceramic HTSC is not caused by electron-phonon interactions. This argument completely ignores the fact that doping is required to render ceramic materials metallic and superconductive, whereas substitutional doping of $\mathrm{MgB}_{2}$ drastically depresses $T_{c}$. From these contrary chemical doping trends it is already obvious that something drastically different from crystalline superconductivity must be happening to cause ceramic HTSC. These drastic differences are likely to be caused by the strong disorder introduced by the dopants. Perhaps the most dramatic differences are apparent in the phase diagrams of $T_{c}(x)$ in $\mathrm{Ba}_{1-x} \mathrm{~K}_{x} \mathrm{BiO}_{3}$ compared to $\mathrm{La}_{2-x} \mathrm{Sr}_{x} \mathrm{CuO}_{4}$, as shown in Figure 1. As both compounds are oxides, one might have expected to see similar phase diagrams, but clearly the standard parabolic $T_{c}(x)$ of the cuprates is not seen in the triangular $T_{c}(x)$ of $\mathrm{Ba}_{1-x} \mathrm{~K}_{x} \mathrm{BiO}_{3}$. The latter is well understood: as $x$ decreases, the dopant free charge density decreases, and with it the screening of electronphonon interactions, so $T_{c}(x)$ increases right up to the metal-insulator transition. Our main task for the ceramic superconductors is now to explain the parabolic dependence of $T_{c}(x)$ (which is not difficult in a glassy network model), and to predict $T_{c}{ }^{\max }(x)$ at optimal doping (much harder).

Other examples of $T_{c}$ predictions are the proton orderdisorder transition in high-pressure ice, where the predicted and experimental values are $98 \mathrm{~K}$ and $72 \mathrm{~K}$, respectively (KOH doping) [6], and strained thin-film ferroelectrics, where large shifts in the Curie temperature $T_{c}$ are predicted, and there is a good agreement between theory and experiment after correcting the domain effects [7]. In magnetic materials, the values of $T_{c}$ calculated by self-consistent fields with frozen magnons are in generally good agreement with experiment within about $20 \%$, even for complex ternary alloys with mixed ferro- and antiferromagnetic interactions [8]. All of these successful calculations have occurred in well-ordered crystals; methods based on ideal lattice models thus may not be equally successful for strongly disordered materials like ceramic superconductors.

\section{Exotic Superexchange and Traditional Electron-Phonon Interactions}

Much of the theoretical literature over the last two decades has been devoted to discussing nonphonon models of HTSC. Did Bednorz and Mueller suppose, when they discovered HTSC in the cuprates, that they were also opening what would turn out to be a Pandora's box for theorists? Certainly, by looking for superconductivity in a family of materials known not for metallic, but for antiferromagnetic properties, they were already behaving as contrarians, who worked evenings on this project, as they felt that this research direction would not meet with the approval of their immediate superiors. Had they consulted me, I would have asked them why they expected to find superconductivity in these pseudoperovskites. Their explanation that the strongest electronphonon interactions are found in the best ferroelectrics (like $\mathrm{BaTiO}_{3}$, which has the closely related perovskite structure) would not have satisfied me. The perovskites exhibit many displacive distortions, and a would be metal should be rendered insulating by Jahn-Teller distortions.

In fact, my general argument was correct for almost all such materials, almost all, but with a few crucial exceptions(!) As I later learned, the crystal chemist who discovered $\mathrm{La}_{2} \mathrm{CuO}_{4}$ had made a prescient remark (references for this and many other older papers can be found in my book [9]). He noted that $\mathrm{La}_{2} \mathrm{CuO}_{4}$ was the only cuprate known at that time with an undistorted tetragonal structure. In all other cuprates the lattice planes had buckled due to strong JahnTeller distortions, and these same distortions would suppress metallic conductivity and of course superconductivity. No one could blame theory (or me) for not predicting the rigidity of $\mathrm{CuO}_{2}$ planes, and I was off the hook for the moment, but there were much bigger challenges yet to come.

These new challenges emerged in a way that took me even more by surprise than the Bednorz-Mueller discovery. It had been known for decades that superconductivity and magnetism are incompatible, because electron-spin scattering breaks Cooper pairs. There are elaborate manybody ways of deriving this result (based on four-particle scattering diagrams called plaquettes), but the simplest way is to invoke Helmholtz' theorem, which states that any vector field can be decomposed into complementary parts: solenoidal (magnetic) and irrotational (extensive, in other words, superconductive). This is, in fact, a "poor man's" way of deriving the Meissner effect.

Soon phase diagrams appeared that showed that as the doping level increased, magnetism faded and superconductivity appeared, just as one would have expected. However, even before phase diagrams became available, theorists had jumped in with explanations, and the first explanation was not the most natural one, but the most surprising one, even more surprising than the discovery itself. Anderson proposed that HTSC was caused not by phonons, but by superexchange between spins(!), a subject that he had discussed in 1950 
for insulators. It seems that he had long hoped to find superexchange in metals, and it appeared to him that HTSC were just what he had been looking for. He termed his new mechanism "RVB" and it soon became by far his most cited paper ( $\sim 5000$ citations so far). Anderson is still advocating RVB [10] and that HTSC was caused not by phonons, but by superexchange between spins(!), and he has persuaded many distinguished theorists to join him [11]. His example has stimulated many frivolous theories.

Of course, not everyone was in love with superexchange. Sir Neville Mott had always championed a common-sense approach (today known more popularly by the computer scientists' acronym KISS), and initially he suggested that HTSC could be caused by a mixture of electron and spin interactions. However, he soon abandoned spin altogether, and turned to "bipolarons" by which he meant Cooper pairs formed by very strong and very localized electronphonon interactions $[12,13]$. However, while there is no doubt that very localized electron-phonon interactions exist at dopants (such as interstitial O) in these materials, it is not easy, especially with such large unit cells and so many normal modes, to distinguish experimentally such dopant interactions from host Jahn-Teller distortions. Indeed, even in the old intermetallic "high temperature" superconductors, with $T_{c} \sim 20+K$, it was often Jahn-Teller distortions (or other lattice instabilities) that had ultimately limited $T_{c}$ [9], while magnetic interactions, even with small amounts of magnetic impurities, quickly destroyed superconductivity. T. D. Lee also suggested that HTSC might be more like BoseEinstein condensation, and there are still papers on HTSC using the B-E approach. In my opinion, this approach is insufficiently material-specific, and does not identify the key aspects (like the rigidity of $\mathrm{CuO}_{2}$ planes) that make the cuprates special.

From the point of view of materials science, what was special about the cuprates was (and still is) that they are at the cutting edge (sometimes called the bleeding edge) of new materials: not only are they complex chemically, but also oxides per se previously enjoyed a very poor reputation among crystallographers: the samples were often oxygendeficient, and the sample quality was often so poor that $\mathrm{X}$ ray structural determinations did not meet the standards required for publication in archival diffraction journals. It seemed to me [14] that these problems suggested that these strongly disordered materials should be regarded as mechanically marginally stable (MMS). MMS is apparently unapproachable theoretically; the reason is that too little is known about interatomic forces, and with many atoms/(unit cell), even small errors in these will cause the dynamical matrix to produce negative values of $\omega_{\alpha}^{2}(q)$ for some $q$ and some mode $\alpha$. The current state of the art (2009) is that MMS in ferroelastic and ferroelectric $\mathrm{BaTiO}_{3}$ (but not $\mathrm{La}_{2} \mathrm{CuO}_{4}$ ) has been solved by brute-force first-principles pseudopotential calculations. However, even the host compound $\mathrm{La}_{2} \mathrm{CuO}_{4}$ is a very long way from $\mathrm{La}_{2-x} \mathrm{Sr}_{x} \mathrm{CuO}_{4}$ (large lattice distortions near dopants are always ignored, as they are a large problem for brute-force approaches, but as we will see, there are other ways to deal with them).
When $T_{c}$ is calculated for HTSC using crystalline continuum metallic $\left(\lambda\left\langle\omega^{2}\right\rangle\right.$ or Eliashberg) rules, not only are the predicted values too low but also the chemical trends are wrong (with some fudging of the adjustable Coulomb repulsion parameter $\mu^{*}, T_{c}$ in $\mathrm{La}_{2-x} \mathrm{Sr}_{x} \mathrm{CuO}_{4}$ may be brought close to experiment $\left(x_{c}=0.15, T_{c}=38 \mathrm{~K}\right)$, but the same value of $\mu^{*}$ in $\mathrm{YBa}_{2} \mathrm{Cu}_{3} \mathrm{O}_{7-x}$ gives $T_{c}<1 \mathrm{~K}$, a failure compared to experiment $\left(x_{c}=0.1, T_{c}=90 \mathrm{~K}\right)[15]$. The feature that distinguishes ceramic HTSC from metals is their strong disorder, as reflected in complex patterns of nanodomains on a length scale $\sim 3 \mathrm{~nm}[16,17]$. In the presence of strong disorder, superconductivity may become molecular in character $[12,13]$, in which case $T_{c}$ could be as high as $380 \mathrm{~K}$ [18]. Unfortunately, this upper bound is too weak, and is unlikely to be reached, because in the presence of strong electron-phonon interactions the metallic band at $\mathrm{E}_{\mathrm{F}}$ will be split by a Jahn-Teller effect (e.g., monovalent dopants could form electrically inactive dimers); worse still, the entire compound may not be dopable, or may phase-separate.

\section{Chemical Factors: Size, Apical Oxygens, Electronegativity, and Valence}

A different and more global approach to the exponential complexity associated with strong disorder relies on traditional analysis of chemical factors (valid in crystals, molecules, and glasses) [19] to analyze trends in $T_{c}$. Some have argued that cuprate superconductivity must be localized in the $\mathrm{CuO}_{2}$ planes [20], but if this were the case, the maximum $T_{c}$ 's in each material family, $T_{c}{ }^{\max }$, would be nearly constant, much like the planar lattice constant. This is far from being the case. As one can easily see from structural systematics [19], the central problem in ceramic superconductors lies in making a ceramic conducting by heavy doping. Light doping (as in semiconductors) is not enough because as the ceramic dielectric constant is small, the dopant orbitals are also small, and these will not overlap unless the dopant level is high. Maximum doping levels in rigid semiconductors are typically $<1 \%$, so something special is required to support the high doping levels found in ceramic superconductors and still avoid phase separation.

In cuprates, the special factor is the exceptional rigidity of $\mathrm{CuO}_{2}$ planes (see Section 2), which allows the intervening insulating layers to be soft and flexible. Because core repulsive potentials are "hard" it is then relatively easy to dope the soft insulating layers. Note that normally one thinks of metals as soft, and ionic insulators as hard. The cuprates and a few other layered ceramics reverse the normal ordering, and this is what makes it possible for them to be HTSC. There are no mysterious superexchange interactions between spins; most of the antiferromagnetic regions have disappeared by the time the doping is large enough for the materials to be metallic. The pseudogaps are still present, and they play a necessary role, but whether or not they are caused by magnetic interactions (unlikely) or charge density waves (or Jahn-Teller distortions) (probably) is incidental. The highest $T_{c}$, reached at optimal doping in a given alloy will depend on the dopant configuration relative to the regions occupied 
by the pseudogaps. That, in turn, will depend on the relative sizes of the ions, a packing (actually, a dynamical packing) question that is well beyond the reach of present and probably any future theory. As we will see in the following section, with a sufficiently large data base this difficulty can be handled by advanced statistical methods.

Kamimura recognized that the $\mathrm{CuO}_{2}$ planes alone could not explain HTSC, no matter how exotic the interactions within them, and he suggested that apical oxygens could play an important role [21]; several succeeding "apical oxygen" papers also proved quite popular $[22,23]$. There have always been strong indications that apical oxygens are coupled to interlayer dopants (especially interstitial oxygen, which is close to apical oxygens and can form dynamical vibrational bands with them) based on chemical trends between apical oxygen bond lengths, ordering and $T_{c}$ [24], and we will see further evidence of such correlations later. However, specific apical oxygen models always introduced atomic orbitals and their overlaps, which generates many parameters to fit one observable, a situation that always leads to "excellent" agreement between "predicted" and experimental values. In fact, the most popular model [23] is based on the difference between the Madelung potentials at the planar and apical oxygen sites, calculated with an artistic point ion model where most of the many charges in the unit cell are freely "assigned" (e.g., although $\mathrm{O}$ is usually $2-$, in some cases it is "assigned" a charge of $1+$, and many liberties are taken with "assigned" cationic charges). A parameterized discussion of correlations between apical oxygen bond lengths $d_{a}$ and $T_{c}$ [25] gave plausible trends, but it covered only a few cases with no predictions of $T_{c}$, showing the limitations of $d_{a}$ as a configuration coordinate. More generally, one should not use extensively parameterized atomic models $\left(\mathrm{CuO}_{2}\right.$ planes + apical oxygens) to discuss differences between pseudogapped regions and superconductive regions, as this difference is almost surely due to different dopant distributions [26].

When one considers seven different structural factors, including apical oxygen bond lengths, and correlates them with $T_{c}$, one finds that the obvious ionic variable, the average over all host atoms of the difference between cation and anion electronegativities $(\langle\Delta X\rangle)$, gives the best fit to $T_{c}[27]$. (This is the ionic analogue of the covalent molecular model [14].) Bearing in mind that different dynamical packings can be used to maximize $T_{c}$, we can now test the validity of $\langle\Delta X\rangle$ as a coupling parameter by plotting $T_{c}{ }^{\max }(\langle\Delta X\rangle)$. The results are quite disappointing, as they yield a scatter-shot plot [28], which means that the traditional chemical approach is too simple to explain HTSC (the reason is that to produce a strong electron-phonon interaction, we need a soft lattice, and soft lattices will be found near a covalent-ionic-metallic triple point, which will not depend on $\langle\Delta X\rangle$ alone). It is just at this point, when nothing seems to work, that we can find the answer, using only the elements discussed so far.

\section{Successful Prediction of $T_{c}^{\max }$}

We begin by realizing that volume factors although they are not known in detail, can be included implicitly in the analysis by focusing initially not on $T_{c}(Y)$, but rather on the largest transition temperatures $T_{c}{ }^{\max }(Y)$, where $Y$ is any other chemical factor. In other words, if the volume factor has already been optimized, then the material in question will have $T_{c}=T_{c}{ }^{\max }(Y)$. For those familiar with Bayesian probability, this approach is readily recognized: Bayesian probability interprets the concept of probability as a contingent "measure of a state of knowledge" and not as a frequency in orthodox statistics (further details can be found from your browser). Bayesian methods can be extremely effective, but the Bayesian filter requires a large data base. In what follows, the basic Bayesian conclusions are tested by employing the full ceramic HTSC data base, which has now grown quite large, including ceramics based on metallic layers other than $\mathrm{CuO}_{2}$ planes and apical ions other than oxygen.

At this point there are only two chemical configuration coordinates remaining: electron/atom (e/a) ratio (which determines the Fermi line of a two-dimensional metal), and valence, which apparently is relevant for perovskites and pseudoperovskites, where the average coordination number is far lower than in metals. In a continuum model the e/a ratio would be the relevant one, while in a valence bond network model valence is the key coordinate; this coordinate can compete with structural coordination numbers [14]. The latter are much the same for most cuprates, and are unlikely candidates for $Y$. Moreover, decades of research on a parallel problem, network glasses, have shown (particularly when comparing silicate and chalcogenide alloy glasses) that the MMS of such densely packed glasses (which permits them to avoid crystallization) depends primarily on the number of Pauling resonating valence bonds $R$ (e.g., in $\mathrm{NaCl}$ there is one Pauling resonating valence bond/ion, although the coordination numbers are six) [29]. The value of $R$ for $\mathrm{Cu}$ in the cuprates is 2 . There are similarly obvious rules for $R$ for other elements (including those with mixed valence, $\mathrm{Tl},+1,+3$, and $\mathrm{Bi},+3,+5)[30]$, and of course, $R(\mathrm{O})=2$, while $\mathrm{e} / \mathrm{a}(\mathrm{O})=6$. The conclusion (also taking into account many results for network glasses) is that while $R$ is a good coordinate for ionic and covalent molecules, it is a very accurate configuration coordinate for strongly disordered networks, even in the presence of lone pair interactions [28]. The valence bonds that one is counting determine the MMS of the network, while the dopants provide the metallic carriers. Of course, in metallic superconductors, e/a is the relevant chemical factor, so by replacing e/a with $R$, we have not only identified the central difference between metallic and ceramic superconductivity, but we have done so quantitatively.

\section{MMS and Coarse Graining}

The question remains of how valence or $R$ is to be averaged over the many atoms in the unit cell: should the rigid metallic planes be weighted more heavily, or the soft layers between them, where the dopants are. Again decades of research on network glasses have shown that the best weighting is the simplest, that is, equal weighting $(\langle R\rangle)$ for all atoms 
provides the best description of glass-forming ability and the glass transition, because the overall network is mechanically marginally stable (MMS). As discussed earlier, shell-model calculations [31] of phonon spectra in HTSC are generally unable to predict very soft modes, especially those associated with dopants. Quite revealing is the fact that the fitted effective charges for "soft layer" rare earth cations in shell models of LSCO and YBCO phonon spectra derived from neutron scattering were inexplicably negative [32]. Neutron scattering has successfully identified Jahn-Teller distortions associated with high-frequency optical modes, and these set the overall scale [30] for $T_{c}{ }^{\max }$, but it is unable to resolve very soft modes [33].

Although MMS is concealed from experiment and theory in HTSC, the simple and universal assumption of equal weighting of all atoms is fully effective. In retrospect, it is easy to see why equal weighting works so well. HTSC exhibits nanodomains spanning $\sim 10$ unit cells $[16,17]$, so the soft modes implied by MMS are spread not only over a single unit cell, but in fact over many unit cells (coarse graining), all of which are sufficiently disordered by dopants and pseudogap instabilities that equal weighting within a unit cell becomes almost as accurate as in fully homogeneous network glasses.

Guided by these considerations, which are general, specific, simple, and free of adjustable parameters, we plotted $T_{c}{ }^{\max }(\langle R\rangle)$, with the results shown in Figure 3. It is important to understand how the predictive dotted line was drawn. First, note that all the HTSC with $R \geq 2$ are well known, and in fact were discovered chronologically with decreasing $\langle R\rangle$, starting with $\mathrm{Ba}_{1-x} \mathrm{~K}_{x} \mathrm{BiO}_{3}$ (a cubic perovskite, with a phase diagram quite different from the cuprates, and only hints of an emerging nanodomain structure [30], but it still fits nicely on the smooth dotted line). Secondly, if one knows only the ascending curve for $\langle R\rangle \geq 2$, one might reasonably extrapolate it to very large $T_{c}{ }^{\max }(\langle R\rangle)$ for $\langle R\rangle<2$. When the predictive dotted line was drawn initially, only one point was known for $\langle R\rangle<2$ that for the oxyhalide $\mathrm{NCCOC}\left(\mathrm{Na}_{x} \mathrm{Ca}_{2-x-y} \mathrm{CuO}_{2} \mathrm{Cl}_{2}\right)$ with $\mathrm{Na}$ (not O!) dopants, an apical $\mathrm{Cl}$ (not $\mathrm{O}$ !) and $T_{c} \sim$ $40 \mathrm{~K}$. There is no phenomenological reason to suppose that this value of $T_{c}$ represents a realization of $T_{c}$ max, that is, that $T_{c} \sim 40 \mathrm{~K}=T_{c}{ }^{\max }$. Nevertheless, one can note that $T_{c}$ (NCCOC) is approximately the mirror image of $T_{c}$ (LSCO) about $\langle R\rangle=2$, so on the basis of percolative symmetry it seemed natural to draw the predictive line as shown. Later, $\mathrm{Ba}_{2} \mathrm{Ca}_{2} \mathrm{Cu}_{3} \mathrm{O}_{6} \mathrm{~F}_{2}$ (apical $\mathrm{F}$ ) was reported to have $T_{c}=55 \mathrm{~K}$, which is comfortably below $T_{c}{ }^{\max }=78 \mathrm{~K}$ on the predicted line, so this point was added to the published Figure [30] as its first "predictive" success, assuming that this value still did not represent a realization of $T_{c}$ max . Finally postdated improved sample preparation $\left[34\right.$ ] gave $T_{c}(\mathrm{BCCOF})=76 \mathrm{~K}$, reducing the discrepancy between the predicted value of $T_{c}{ }^{\max }$ and experimental value of $T_{c}$ for BCCOF from $23 \mathrm{~K}$ to $2 \mathrm{~K}$. This is a very convincing second (and predated) predictive theoretical success, especially considering that neither NCCOC nor BCCOF contain apical O, which means that the homology is unexpectedly general. Of course, further large increases in $T_{c}$ (NCCOC or BCCOF) by $20 \mathrm{~K}$ would be sufficient to falsify the theory, although even then

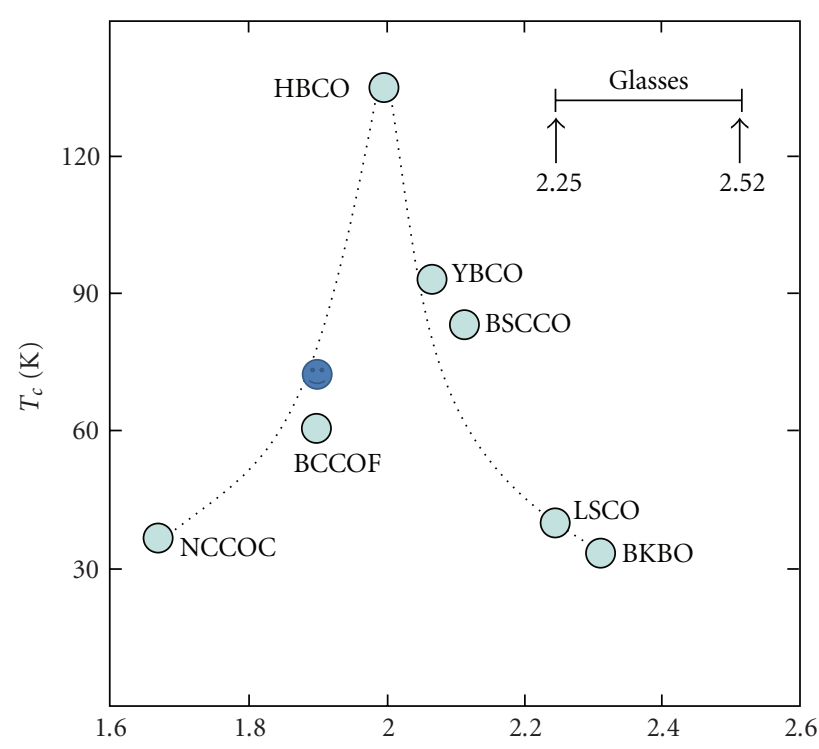

$\langle R\rangle$

Figure 2: The master function for $\operatorname{HTSC}, T_{c}{ }^{\max }(\langle R\rangle)$, provides a least upper bound for bulk layered superconductors, and is believed to be accurate to $10 \mathrm{~K}$. This function is based on the zigzag percolative model for self-organized HTSC dopant networks $[28,30]$. The original figure [30] has been modified to include the most recent data [34] on BCCOF; the acronyms are as in [30]. Covalent network glasses [20] are centered on $\langle R\rangle=2.4$, but the stabilizing effect of the rigid $\mathrm{CuO}_{2}$ planes shifts the cuprate center to $\langle R\rangle=2$.

it would be better than the only available alternatives which predict $T_{c}{ }^{\max }$ higher by as much as $200 \mathrm{~K}$.

The analysis given here is based on minimal logical and statistical considerations, but the results were actually derived from a physical model that the author first proposed 20 years ago [35]. The zigzag self-organized percolative model (ZZIP) has many attractive features: for instance, the energy scale for $T_{c}$ max , which is merely set empirically in Figure 3, is apparently set for $\langle R\rangle \geq 2$ by the Jahn-Teller shift in the (100) LO phonon energy (there are no oxyhalide data for $\langle R\rangle<2$ ), as shown by Figure 3(b) in [30], which suggests a close dopant-mediated relation between the soft acoustic and optic modes. Substantial evidence shows that percolative filaments are formed at high temperatures and account for many features of transport up to at least $300 \mathrm{~K}$ [30]. At present there exists no alternative model that can predict $T_{c}{ }^{\max }$ in HTSC.

\section{Cuprate-Like Superconductivity}

There are several new marginally stable families of layered crystals that exhibit many similarities to the cuprates: there are many atoms per unit cell, with displacive lattice instabilities, vicinal antiferromagnetic phases, and so forth, including ionic $\mathrm{Li}_{x} \mathrm{ZrNCl}$ [36] and the rapidly growing covalent superfamily based on FeAs, such as $\mathrm{LaFeAsO}_{1-x} \mathrm{~F}_{x}$ [37]. Are these similarities accidental, or can the percolative cuprate 


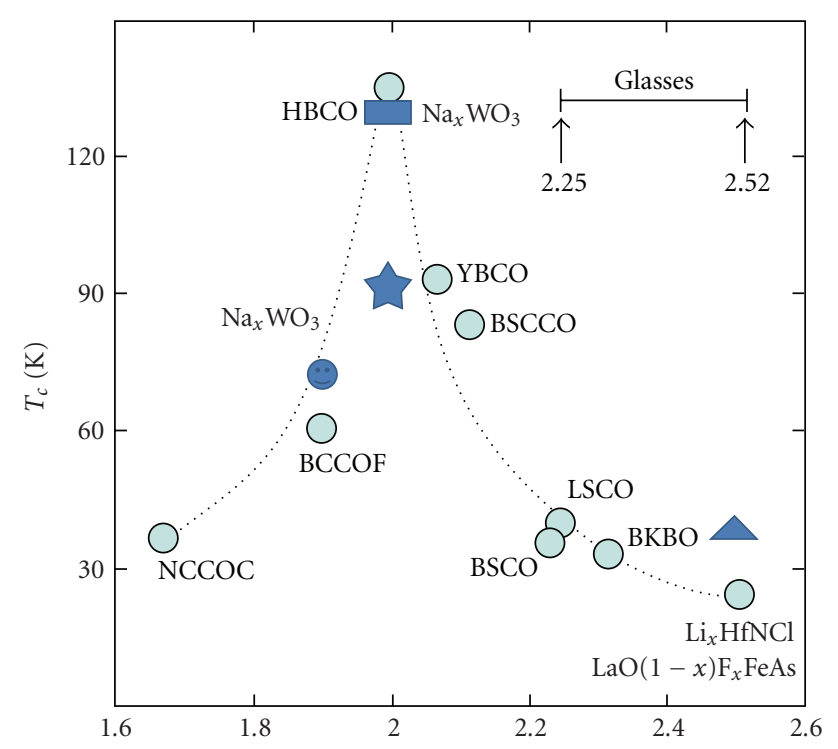

$\langle R\rangle$

Figure 3: As in Figure 2, but now the noncuprate data have been added, so the Figure has become rather "busy."

ionic superfamily model explain HTSC in these noncupratelayered materials with no additional assumptions? In fact, the percolative model easily explains the similarities, by bringing these new materials into the general framework of selforganized networks. This has already been done for ionic $\mathrm{Li}_{\mathrm{x}}(\mathrm{Zr}, \mathrm{Hf}) \mathrm{NCl}\left(T_{c} \sim 15 \mathrm{~K}-25 \mathrm{~K}\right)$ [38], so now a similar discussion is given here for the covalent $\mathrm{LaFeAsO}_{1-x} \mathrm{~F}_{x}$ superfamily $\left(T_{c} \sim 26 \mathrm{~K}-43 \mathrm{~K}\right)$ [37], which is much larger and the subject of hundreds of recent studies.

Because these materials are mechanically only marginally stable, they are strongly disordered when doped, and are generally far from optimized with respect to HTSC. Marginal lattice stability determines the overall scale for $T_{c}$, as the phonon energy shift measured by neutron scattering associated with Jahn-Teller doubling of the unit cell of LO phonons correlates linearly with $T_{c}{ }^{\max }$ in the cuprates [30]; of course, spin and antiferromagnetic exchange show no such scaling, and are irrelevant. The least upper bound for $T_{c}$, called $T_{c}{ }^{\max }$, has a strongly percolative character, as it peaks exponentially at $\langle R\rangle=2$, where $\langle R\rangle$ is the average valence number of all the atoms $[28,30]$.

The master function $T_{c}{ }^{\max }(\langle R\rangle)$ is shown in Figure 2 for the cuprates; the peak at $\langle R\rangle=2$ has a cut-off exponential character. The point for ionic $\mathrm{Li}_{\mathrm{x}} \mathrm{HfNCl}$ was discussed previously [38], and we now discuss the points for the LaFeAsO ${ }_{1-x} \mathrm{~F}_{x}$ family $\left(T_{c} \sim 26 \mathrm{~K}-43 \mathrm{~K}\right)$. Here $R(\mathrm{Fe})=2$, just as for $\mathrm{Cu}$, because $\mathrm{Fe}$ is in a $2+$ valence state $[39,40]$ in a virtual crystal model. This band model also shows the beginnings of self-organization, in that the average height $h$ of As is found to shift with $x$ [41]; had the calculation been carried out with a large supercell centered on an $\mathrm{F}$ dopant?, $h$ (As) would have varied with distance from $\mathrm{F}$. This virtual crystal relaxation effect alone shows that $\mathrm{N}\left(\mathrm{E}_{\mathrm{F}}\right)$ varies slowly and smoothly with doping (which means that the apparent Fermi planar line becomes very broad near a critical point), and hence effective medium models cannot explain HTSC. However, from this one should not conclude that electron-phonon interactions do not cause HTSC, as these interactions (not superexchange!) do set the overall energy scale [30] through Jahn-Teller distortions on and near percolative paths, and these distortions are especially large near dopants.

When one calculates $\langle R\rangle$ for undoped $\mathrm{LaFeAsO}$, one obtains $\langle R\rangle=2.5$, which places $\mathrm{LaFeAsO}_{1-x} \mathrm{~F}_{x}\left(T_{c}=26 \mathrm{~K}\right)$ very close to $\mathrm{Li}_{x} \mathrm{ZrNCl}$ on the master curve of Figure 2, so that the theory appears to succeed effortlessly. However, $T_{c}$ is maximized at $43 \mathrm{~K}$ for pressures near $4 \mathrm{GPA}$ [42], an increase of $60 \%$, which is much more than is seen in cuprates, and exceeds the upper bound of the master curve. Does this falsify the theory? No, because the equilibrium cuprate master function $T_{c}{ }^{\max }(\langle R\rangle)$ remains valid for the $\mathrm{Li}_{x} \mathrm{ZrNCl}$ and $\mathrm{LaFeAsO}_{1-x} \mathrm{~F}_{x}$ families, but the pressure dependence in the latter family is larger than in the cuprates, as Fe-As bonding is more covalent than largely ionic $\mathrm{Cu}-\mathrm{O}$ bonding [42], due to the larger Pauling electronegativity $X$ differences $(X(\mathrm{Fe})=1.8, X(\mathrm{As})=2.0, X(\mathrm{Cu})=1.9, X(\mathrm{O})=3.5))$ in the cuprates. Covalent bonding also seems to limit the range of $\langle R\rangle$ for which stable crystals can form; thus the smallest value of $\langle R\rangle$ for the FeAs family seems to be near 2.3 in $\left(\mathrm{Ba}_{0.55} \mathrm{~K}_{0.45}\right) \mathrm{Fe}_{2} \mathrm{As}_{2}[43]$.

It is striking that this covalent FeAs stability range of $2.3<\langle R\rangle<2.5$ is very similar to the range $2.25<\langle R\rangle<$ 2.52 for stress-free covalent glasses previously shown $[28,30]$ in Figure 2. (Again this is a rather remarkably successful predated prediction.) Thus the covalent instabilities of the host lattice, especially the reduced rigidity of the FeAs plane relative to the $\mathrm{CuO}_{2}$ plane, are the factor that shifts the value of $\langle R\rangle$ at which $T_{c}{ }^{\max }$ peaks from $\langle R\rangle_{\max }=2.0$ in the ionic cuprates to $\langle R\rangle_{\max }=2.5$ in the $\mathrm{LaFeAsO}_{1-x} \mathrm{~F}_{x}$ family. In both cases,

$$
\langle R\rangle_{\max }=R(\text { metallic plane }),
$$

so that the average connectivity of the undoped insulating plane is matched to that of the metallic plane. Apparently this hidden topological layer symmetry (1) determines the maximum electron-phonon interactions in marginally stable layered pseudoperovskites.

\section{Surfaces and Interfaces}

The percolative master function $T_{c}{ }^{\max }(\langle R\rangle)$ is determined from bulk data on layered crystals, so one can ask whether or not this function can explain trends in $T_{c}{ }^{\max }$ at surfaces and layer interfaces. Determining $\langle R\rangle$ at surfaces and interfaces is much more difficult than in the bulk, where it is natural to average $R$ over all atoms, as the self-organized structure is marginally stable overall, and soft modes that are critically bound to percolative superconductive paths should have long wave lengths, as $T_{c}{ }^{\max }$ is still small compared to the melting temperature. However, in layered thin crystalline films with epitaxial interfaces or in clusters at doped free surfaces, similar percolative behavior is expected. This turns out to be 
the case for the $\mathrm{La}_{2} \mathrm{CuO}_{4}-\mathrm{La}_{2-x} \mathrm{Sr}_{x} \mathrm{CuO}_{4}(x=0.45)$ interface, where $T_{c} \sim 50 \mathrm{~K}$, after enhancement by exposure to ozone from $\sim 30 \mathrm{~K}$ [44]. The giant ozone enhancement is readily explained by the addition of oxygen dopants, absorbed by $\mathrm{La}_{2} \mathrm{CuO}_{4}$ to give $\mathrm{La}_{2} \mathrm{CuO}_{4+\delta}$ with $\delta \sim 0.15$. However, the maximum $T_{c}$ obtainable in this way is $\sim 30 \mathrm{~K}$ for both layers separately, apparently producing a mystery.

Let us look at this mystery with the master curve: $\langle R\rangle$ $=16 / 7$ in $\mathrm{LCO}=2.28,=(16-x) / 7$ in $\mathrm{La}_{(2-x)}=\mathrm{Sr}_{x} \mathrm{CuO}_{4}$, so with $x($ or $\delta)=0.15,\langle R\rangle=2.26$, and with $x=0.45,\langle R\rangle$ $=2.22$. This would give a decrease in $\langle R\rangle$ between $x=0.15$ and $x=0.45$ of 0.04 , and so we get something like $T_{c}=35 \mathrm{~K}$ $+(0.04 / 0.28)(150-35) \mathrm{K}=50 \mathrm{~K}$. Of course, this is just a plausible guess at the interfacial structure, but the master function has given the trend correctly, not only qualitatively but also semiquantitatively (something no other theory has been able to do: virtual crystal theories predict $T_{c}<1 \mathrm{~K}$, from which it has often been erroneously concluded that electronphonon interactions do not cause HTSC!).

Now we turn to a much more difficult problem, for which the data base is small, but still robust: a surface monolayer of $\mathrm{A}_{x} \mathrm{WO}_{3}$, where $A$ is an alkali metal ( $\mathrm{Na}[45]$ or Cs [46]). While bulk $\mathrm{Na}_{x} \mathrm{WO}_{3}$ exhibits superconductivity only near $1 \mathrm{~K}$, here for $\mathrm{Na}$ superconductivity appears around $100 \mathrm{~K}$; for Cs there are two phase transitions, a bulk one with lower $T_{c}$ at higher doping, and a re-entrant percolative one with higher $T_{c}$ at lower doping. Moreover, $\mathrm{Na}-$ and $\mathrm{Li}-$ (but not $\mathrm{K}-$ ) doped surfaces of nanoclusters of $\mathrm{WO}_{3}$ embedded in a variety of nanoporous hosts (carbon inverse opal, carbon nanotube paper, or platinum sponge) show diamagnetic anomalies with an onset $T$ of $130 \mathrm{~K}$ [47]. Note that $\mathrm{WO}_{3}$ (with its simpler unit cell, subject only to Jahn-Teller distortions) itself is nonmagnetic, as is another $\operatorname{HTSC}\left(\mathrm{BKBO},(\mathrm{Ba}, \mathrm{K}) \mathrm{BiO}_{3}\right)$.

These data can be combined with the master function $T_{c}{ }^{\max }(\langle R\rangle)$ to construct a model of percolative selforganization at surfaces. In bulk $\mathrm{WO}_{3}$ the valence of $W$ is 6 , and $\langle R\rangle=3.0$, far to the right on the master function $T_{c}^{\max }(\langle R\rangle)$, where $T_{c}{ }^{\max }(3)<5 \mathrm{~K}$. Near the surface the valence of $W$ could be 2 (just as with $\mathrm{Cu}$ in the cuprates, and $\mathrm{Fe}$ in the FeAs compounds). To explain $T_{c} \sim 130 \mathrm{~K}$, one must assume $\langle R\rangle=2$. A percolative $\mathrm{W}_{\mathrm{s}} \mathrm{O}$ surface chain then has $\langle R\rangle=2$. These surface chains are entropically broken into stress-relieving fragments. Intercalated Li or $\mathrm{Na}$ ions connect the chain fragments, thereby increasing their conductivity and their screening of internal ionic fields, just as in the bulk percolative model. The embedded clusters are not connected, so the result is "localized nonpercolative superconductivity" still with $\langle R\rangle=2$ [48]. In the free surface case $[45,46]$, thermal fluctuations disrupt superconductivity above $100 \mathrm{~K}$. Both of these $\langle R\rangle=2$ points are shown in Figure 2. Considering the rapid progress in nanoscience, it may be possible to obtain similar pairs of percolative and cluster points for other HTSC.

Finally, self-organized percolation enables us to understand how cointercalation of organic molecules $\mathrm{M}$ with $\mathrm{Li}$ in ionic $\mathrm{Li}_{\mathrm{x}} \mathrm{M}_{\mathrm{y}} \mathrm{HfNCl}$ can uniformly enhance $T_{c}(x)$ by up to $30 \%$, over a wide range $0.15 \leq x \leq 0.50$, even though the average interlayer spacing $d$ varies by as much as $30 \%$ [49]. The intercalated organic molecules reduce the dielectric screening of $\mathrm{Li}$-centered electron-phonon interactions by other Li ions. Note that the nominal concentration $x$ of the Li ions may refer only to patches that strongly diffract; the superconductive paths may pass through patches with a concentration $x_{0}$ different from $x$, explaining why $T_{c}$ is apparently constant over a wide range of $x$. Only a few percolative paths are required to exclude Abrikosov vortices and produce HTSC; this is why $T_{c}$ often increases when the average density of states $\mathrm{N}\left(\mathrm{E}_{\mathrm{F}}\right)$ decreases (e.g., in the cuprates compared to the covalent FeAs family, or the ionic $\mathrm{Li}_{x} \mathrm{M}_{y} \mathrm{HfNCl}$ family [38]). This is yet another example of the paradoxes generated by attempting to understand a selforganized percolative phase in terms of continuum concepts such as Bloch-wave Fermi surfaces, modulation doping, or plasmon waves. Note that these points have often been made in earlier papers on the topological percolative model, long before any of these new materials were discovered.

\section{Mapping ZZI Percolative Paths}

For most of the last two decades experiment has provided largely circumstantial support for the zigzag interlayer percolative (ZZIP) network model shown in Figure 4, but recently more direct evidence has appeared. The model has two key elements: the dopants, often interstitial oxygen, whose positions are not easily determined, and the zigzag network paths themselves. The zigzag network paths are associated with strong electron-phonon interactions, which are especially strong for interlayer displacements. This has enabled the zig and the zag of the paths to be identified by a spectacular combination of time-resolved electron diffraction [50], for the $c$-axis zag component of the paths, and anisotropically strong kinks in quasiparticle dispersion observed by angle-resolved photoemission [51], for the ab planar zig component of the paths. The data show that the $c$-axis $\mathrm{Cu}-\mathrm{O}$ zag component has faster dynamics when the femtosecond pulse probe is polarized along the planar $\mathrm{Cu}-\mathrm{O}$ zig component, to which it can be coupled through planar $\mathrm{Cu}-\mathrm{O}$ bond buckling. It is difficult to understand these correlations unless the $c$-axis zag component is actually topologically connected to the planar zig component. One might argue that such a connection occurs only at low temperatures, and would be ineffective at the high excitation energies of the electron diffraction experiment, but this is not correct. High-temperature transport data show that the self-organization (hard wiring) of the ZZIP network begins already at annealing temperatures [30]; the results are fully consistent with the zigzag network model discussed in dozens of papers over the last 20 years. There remains one puzzling aspect: anisotropy is observed in both Bi2212 and Bi2223 by ARPES, but only in the former by electron diffraction. The extra layer in the latter would not appear to erase the planar anisotropy, but it is possible that Bi2223 has a high density of stacking faults. Depending on the geometry of the percolative paths, such faults could erase the observed anisotropy by scattering percolative carriers.

While we are on the subject of percolation versus continuum models, it is important to realize that while the presence of self-organized percolative paths introduces 

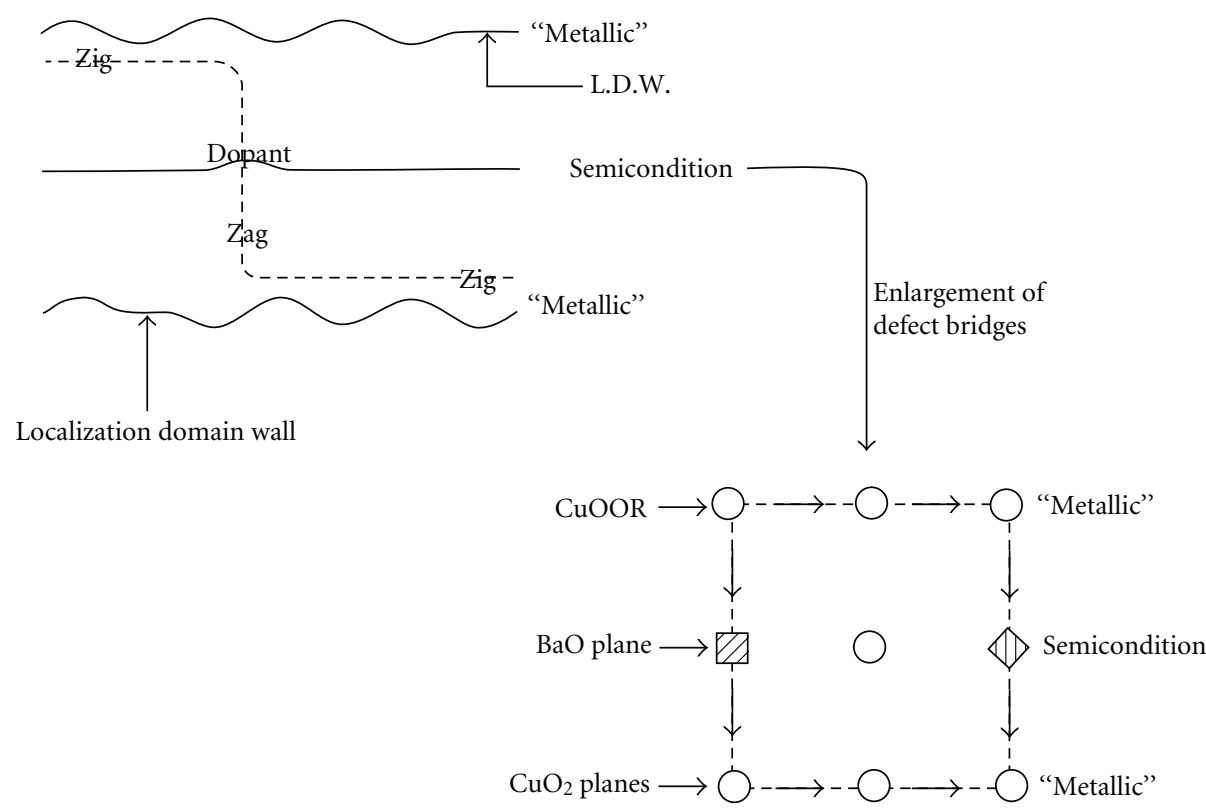

O vacancy

YBa defects

FIGURE 4: The original ZZIP model, reprinted from [35]. Parts of the original ZZIP path are now labeled "zig” or "zag.” In 1989 it was not clear what the interlayer dopants were and they were simply called defects, but it is now clear that they are usually interstitial oxygen atoms, except in $\mathrm{La}_{2-x} \mathrm{Sr}_{x} \mathrm{CuO}_{4}$, where they are the substitutional Sr ions.

exponential complexity into the Bohmian wave-packet basis states [52] used to form Cooper pairs, it in no way alters the nature of the attractive electron-phonon interactions responsible for forming the pairs. These two points have often been confused, and the failure of continuum plane wave basis states to describe cuprate HTSC has often been used to argue that electron-phonon interactions do not cause HTSC [11]. Because the fraction of carriers involved in percolative paths is small, many negative indications of strong electron-phonon coupling have been found; for example, bulk phonon softening is so small as to be unobservable by neutron scattering at high energies [33]. Therefore it is gratifying that quasiparticle tunneling across a break junction perpendicular to the superconducting copper oxide planes showed 11 phonon features that match precisely with Raman spectra [53], decisively showing that HTSC is indeed caused by electron-phonon interactions along the $c$-axis, as previously argued from dynamical relaxation experiments [54]. Also the long-expected isotope effect at the phonon kink has been observed by ARPES [55]. The fact that all the $c$-axis phonon bands appear in the break junction experiment, and not just those associated with the $\mathrm{O}$ buckling mode in the $\mathrm{CuO}_{2}$ plane [51], strongly supports the present model of zigzag percolative paths in a marginally stable all-atom network. Finally, it is worth stressing that the phonon features that correlate by far the best with $T_{c}$ are exactly those of the atoms in the soft insulating layer [56, Figures 11 and 12], as assumed in the zigzag model, and not the atoms in the rigid $\mathrm{CuO}_{2}$ layer, as is often wrongly assumed [51].
There is one interesting aspect of the original 1989 ZZIP model that was confirmed experimentally by anomalous X-ray diffraction [57], but the significance of the data was not realized because they were not connected to the theoretical model. Namely, in the model the metallic layer nanodomains are staggered, as this facilitates the construction of filamentary paths that pass through dopants in the nonmetallic layers. The data showed that "in each $\mathrm{CuO}_{2}$ bilayer the flat stripes in a first layer are close to the bent stripes in the second layer" from which it was concluded that such a staggered structure "suppresses the electronic coupling between the two adjacent planes". In the ZZIP model just the opposite is true: the nanodomains are staggered in order to increase the electronic coupling between the two adjacent planes, and this is part of the selforganization of the "hard-wired" filamentary network that is formed at high temperatures (above $300 \mathrm{~K}$ ), as shown by many normal-state transport anomalies, notably the linearity in $T$ of the planar resistivity [7], compared to the $T^{2}$ dependence characteristic of a Fermi liquid. Of course, in a one-dimensional filament, intrafilamentary scattering always gives such a linear dependence.

A few comments are in order regarding the parabolic form of $T_{c}(x)$. This starts for small $x$ when the percolation threshold $x_{1}$ is reached, and it ends for large $x=x_{2}$ when all the isolated percolative paths have lost their separate identities and become metallic bundles. At and above $x=x_{2}$ continuum theory becomes valid, and it immediately shows that $T_{c}(x)$ for $x \geq x_{2}$ is either very small or zero. The reason for this is simple: the charge density is so low that Coulomb 
repulsive interactions outweigh attractive electron-phonon interactions, just as they do in metals like $\mathrm{Na}$ and $\mathrm{Cu}$ itself.

\section{Single-Layer Cuprates: Mechanically Marginally Unstable (MMU)}

The importance of self-organized percolative paths is nicely brought out by the single-layer compound $\mathrm{HgBa}_{2} \mathrm{CuO}_{4+\delta}$ [58], which has $\langle R\rangle=2$. This compound has the largest $T_{c}=$ $95 \mathrm{~K}$ of any single-layer cuprate, but it does not appear in our figures because it is isovalent to $\mathrm{HBCO}\left(\mathrm{HgBa}_{2} \mathrm{Ca}_{2} \mathrm{Cu}_{3} \mathrm{O}_{8}\right)$, which also has $\langle R\rangle=2$ and $T_{c}=T_{c}{ }^{\max }=135 \mathrm{~K}$. There are several interesting aspects to $\mathrm{HgBa}_{2} \mathrm{CuO}_{4+\delta}$ : the $\mathrm{Cu}-$ $\mathrm{O}$ bond lengths (including the apical $\mathrm{O}$ ) are expanded by the $\mathrm{Ba}$ ions (which act as spacers), and the soft $\mathrm{Hg}$ layer is strongly disordered (as expected in the zigzag model). If superconductivity were confined to the $\mathrm{CuO}_{2}$ layer [21] and the apical $\mathrm{O}[22,23]$, this expansion should have greatly depressed $T_{c}$ (because of strengthened Coulomb or ionic interactions), but the contrary occurred. In the 1989 zigzag model [35] the $\mathrm{CuO}_{2}$ layer functions as an electrical connector, while the strong e-p interactions occur in the soft, strongly disordered $\mathrm{Hg}$ layer, so the relatively large $T_{c}=95 \mathrm{~K}$ is a natural feature of the model. The coupling of HTSC to lattice disorder (not order!) is directly and elegantly shown in [59]. Superconductive formation and overlap of zigzag percolative filamentary arrays can increase lattice disorder. When the $\mathrm{CuO}_{2}$ layers were under compressive strain (so that the critical axial zag paths were under tensile strain, and thus more flexible), this is what was observed as $T$ decreased well below $T_{c}$, where the superconductive energy is largest. Very similar effects appear in the FeAs family as well [59]; a more extensive percolative discussion will be given elsewhere.

None of the single-layer cuprates produces a $T_{c}{ }^{\max }(\langle R\rangle)$ for any $\langle R\rangle$. However, the single-layer cuprates form an interesting homology family in their own right; in contrast to Section 5, they can be described as mechanically marginally unstable (MMU). Theorists have long known that individual planes readily buckle. When this happens in the cuprates, the pseudogap charge density wave regions expand, the metallic regions contract, and $n_{s}$ (the density of superconductive carriers) rapidly drops, and with it $T_{c}$ [30]. Thus another factor comes into play, to stabilize the overall MMU network, and retain larger metallic regions. This factor is the purely ionic static Madelung potential (the rigid ion model omits polarization or screening energies, which are important dynamically, but less important statically) [60]. Within the restricted context of single-layer cuprates only, it appears that it is this factor that dominates $T_{c}$. Note, however, that the single-layer cuprate family never contributes to $T_{c}$ max , and therefore this family should be regarded as nonoptimally packed ceramic superconductors (and, therefore, more in need of stabilization). Moreover, the stabilizing space-filling (buttressing) effect of the large $\mathrm{Ba}$ ions in $\mathrm{HgBa}_{2} \mathrm{CuO}_{4+\delta}$ is not accounted for by the Madelung potential, and here $T_{c}$ lies well above the Madelung trend line for the other single-layer cuprates [60]. This is further evidence that the single-layer
MMU cuprates form a different homology family from the multilayer MMS family.

Among the (relatively) low $T_{c}$ ceramic superconductors, $\mathrm{Bi}_{2} \mathrm{Sr}_{2-x} \mathrm{La}_{x} \mathrm{CuO}_{6+\delta}$ has attracted much interest because here it appears that the pseudogap and superconductive gap are best separated $[61,62]$ relative to other micaceous multilayer cuprates. The reason for this is clear: not only is this material MMU, but also the stabilizing ionic factors are weak, reducing the density of superconductive filaments and filament-filament interactions, and producing a more homogeneous superconductive gap that is well separated from a much larger pseudogap.

What else can we learn from the single-layer cuprate MMU family? ( $\mathrm{La}, \mathrm{Sr})_{2} \mathrm{CuO}_{4}$ is a member of this family, and it also falls on the master function $T_{c}{ }^{\max }(\langle R\rangle)$ of MMS superconductors, and so is also an optimally packed ceramic zigzag percolative superconductor. Which factor (MMS or MMU) is more important at the $\mathrm{La}_{2} \mathrm{CuO}_{4}-\mathrm{La}_{2-x} \mathrm{Sr}_{x} \mathrm{CuO}_{4}(x=0.45)$ interface? In the MMU case, we know that the Madelung potential that stabilizes $\mathrm{CuO}_{2}$ planes decreases as $\mathrm{x}$ increases, in accordance with the fact that $T_{c}\left(\mathrm{La}_{2-x} \mathrm{Sr}_{x} \mathrm{CuO}_{4}\right)=0$ $(x=0.45)$. However, the Madelung potential at the $\mathrm{La}_{2} \mathrm{CuO}_{4}-\mathrm{La}_{2-\mathrm{x}} \mathrm{Sr}_{\mathrm{x}} \mathrm{CuO}_{4}(x=0.45)$ interface can be estimated to be that of $\mathrm{La}_{2-x} \mathrm{Sr}_{x} \mathrm{CuO}_{4}(x=0.22)$, so that $T_{c}$ should have decreased from $38 \mathrm{~K}$ at $x=0.15$ to $\sim 30 \mathrm{~K}$. In fact, $T_{c}$ increased from $30 \mathrm{~K}$ to $50 \mathrm{~K}$, which is what the master function $T_{c}{ }^{\max }(\langle R\rangle)$ of MMS superconductors predicts (Section 7). At optimized interfaces, percolative effects dominate, which is what happened with $\mathrm{A}_{x} \mathrm{WO}_{3}$ surface layers (Section 7).

\section{Conclusions}

The present review shows that the original ZZIP model [35, Figure 4] continues to provide an excellent universal guide to the phenomenology of ceramic superconductors. Initially, it explained the large qualitative differences between HTSC in cubic and layered ceramics (Figure 1). It is the only theoretical model so far that has been able predatively to predict $T_{c}(\mathrm{BCCOF}) \sim 75 \mathrm{~K}$, but it also quantitatively predicts superconductive $T_{c}$, even for noncuprate HTSC, starting only from the cuprates, which no other theoretical model has done. The self-organized marginally stable percolative model unexpectedly transcends conventional chemistry (the materials covered include cuprates, tungstates, zirconates, ferrics, arsenides, bismates, oxides, halides, $\mathrm{Li}-, \mathrm{Na}_{-}, \mathrm{Cs}-$, $\mathrm{O}-, \mathrm{F}-, \mathrm{Cl}-\mathrm{doped}$, and the structures include bulk, interface, and nanocluster surfaces); so far as I know, there is no other example in solid phase transitions where theory of any kind (continuum or otherwise) can predict transition temperatures with such universal success. The results make an overwhelming case for a new homology class of glassy network doped superconductors, separate and distinct from continuum metallic superconductors.

As noted previously [30], the theory predicts $T_{c}{ }^{\max }$ with an accuracy of $10 \mathrm{~K}$, compared to a melting point of order $10^{3} \mathrm{~K}$, which is an accuracy of $1 \%$. However, this success contains a subtle aspect, which is that because HTSC is concerned with marginally stable lattices, it is important 
to build this theoretical mechanism into the analysis from the outset (Section 4). This has been done by mapping $T_{c}{ }^{\max }(\langle Y\rangle)$, and examining different choices of $Y$ : only $Y$ $=R$ gives the apparently exponentially resonant peak that is strongly suggestive of percolation.

The theoretical debates stimulated by HTSC have lasted for more than 20 years, and have often severely tried the patience of the physics community. Were they worth it? I believe they were and still are. The basic issue was, and still is, whether it is legitimate to treat a discrete, exponentially complex problem involving strongly disordered, self-organized materials, at the cutting edge of materials science, with the same simplistic continuum methods (including polynomial Hamiltonian algebra and/or Landau order parameters) that students have learned work so well for toy models and some simple crystalline solids. The experimental evidence strongly suggests that this discrete, exponentially complex problem can be solved by focusing on its essential topological features, derived from the network structures of ceramic HTSC [29].

Global topology is still something of an oddity among mathematical disciplines (it is very young, dating from 1877). It is standard among computer scientists, but it is unfamiliar to most chemists and physicists. It is ideally suited to treat complexity problems, including many far afield from science, such as economics $[63,64]$ and difficult problems in biology, such as the network of protein-protein interactions [65]; for pioneering research in NPC computer algorithms addressing exponential complexity R. M. Karp was awarded the 2008 Kyoto Prize. It also transcends the otherwise theoretically insuperable barriers of exponentially complex (NPC, nonpolynomial complete), aperiodic selforganization commonly encountered not only in HTSC but also in protein science $[66,67]$. From sandpiles to proteins, it appears that the best documented examples of self-organized criticality (SOC) are found in network glasses [29] and HTSC [68].

Postscript. The (by now very old) debate over whether HTSC is caused by conventional electron-phonon interactions, or by exotic electron-spin interactions, continues even for the cuprates [69] although it would appear from Raman scattering experiments to have been settled conclusively in favor of phonons $[53,56,70]$. In particular, infrared optical spectra [69] contain a mixture of overlapping superconductive and pseudogap (or Jahn-Teller) features that are not easily separated, even in optimally doped samples. As the infrared spectra are dominated by electric dipole transitions, it is difficult to believe that any of the "coincidences" between isolated neutron magnetic resonances and fictive single optical resonances used to fit [69] these entangled gap spectra are significant, especially as results internally consistent with gap phase diagrams measured by other methods do not appear to have been obtained for underdoped and optimally doped phases. Simpler and better polaronic fits show no correlations between midinfrared peak positions and $T_{c}[71]$.

These infrared data merely reinforce the large qualitative difference between layered ceramics and cubic $\mathrm{Ba}_{1-\mathrm{x}} \mathrm{K}_{\mathrm{x}} \mathrm{BiO}_{3}$ shown also in the phase diagrams of Figure 1; the latter has a normal BCS-type infrared spectrum with $\mathrm{E}_{\mathrm{g}} / \mathrm{k} T_{c}=3.2(3)$
[72]. There have been many studies of the metal-insulator transition at $x=0.3$ in $\mathrm{Ba}_{1-x} \mathrm{~K}_{x} \mathrm{BiO}_{3}$ and these have been interpreted in terms of bipolaron formation [73]. However, a more appealing interpretation of the $\mathrm{Ba}_{1-x} \mathrm{~K}_{x} \mathrm{BiO}_{3}$ metalinsulator transition would be the onset of percolative metallic patches, as the "bipolaronic" infrared dopant peak appears to merge with the CDW band edge at $x=0.3$, where $T_{c}$ is largest. In other words, the two lattice deformations merge to form nonfilamentary continuum superconductive metallic regions in a first order metal-insulator transition.

Application of "first principle" continuum theories to the FeAs family has uniformly led to the conclusion that electron-phonon interactions cannot cause HTSC in this family [39-41, 74, 75]; similar conclusions were reached earlier for the cuprates [15]. The correct conclusion is that continuum (ideal lattice) approximations are invalid because of strong disorder and nanoscale phase separation [76-78], as well as the much more subtle exponentially complex consequences of zigzag self-organized percolation, ZZIP (Section 8). The combination of electron-phonon interactions and ionicity readily explains [79] how superconductivity in the covalent (noncentral interatomic forces) FeAs family can be much more isotropic than in the cuprates [80], yet still retain a lowered dimensionality. Strong disorder explains "hump-dip" pseudogap tunneling features in terms of charge density waves [81].

Probably the most accurate ceramic HTSC phase diagrams are those obtained by studying the planar resistivity $\square_{a b}(T)$, which is most linear in $T$ at and near optimal doping $[30,82]$. The dopant network-forming interactions, although individually weak, are cumulatively very strong because the dielectric energy gained by screening internal electric fields increases as the network conductivity increases due to dopant self-organization. The maximization of the $T$-linearity of $\square_{a b}(T)$ near optimal doping $[30,82]$ was the first normal-state transport anomaly to be discovered, but in the intervening 20 years theory has been unable to derive this maximization, and it seems unlikely that this extremal will ever be derived using conventional polynomial methods (e.g., Landau Fermi liquid theory predicts a $T^{2}$ dependence). This situation is typical of exponentially complex problems which are characteristic of networks, but it can be repaired by carefully designed experiments.

In ceramic HTSC self-organization effects manifest themselves by a sharpening of properties characteristic of networks. An obvious and trivial example of such sharpening is the narrowing of the superconductive transition itself, which can be promoted by annealing; this is a small effect for traditional metallic superconductors, but a large effect in ceramic HTSC. If the maximization of the $T$ linearity of $\square_{a b}(T)$ near optimal doping is indeed caused by network self-organization, then that should be manifested by an increase in $T$-linearity upon annealing. Studies of redistribution of oxygen at a fixed oxygen content in the chain layers of slightly underdoped YBCO with a nonlinear $\square_{a b}(T)$ showed [83] that annealing for 2-8 hours at 400$420 \mathrm{~K}$ does lead to an increase of $T_{c}$ by about $1 \mathrm{~K}$ and a reduction of the nonlinear component in $\square_{a b}(T)$ by $\sim 10$ $15 \%$ just above $T_{c}$. 
The classic work of [82] has recently been extended to high magnetic fields well above $T_{c}$, with spectacular results [84]. It is found that the $\square_{a b}(T)$ regime which is most linear in $T$ occurs in $\mathrm{La}_{2-\mathrm{X}} \mathrm{Sr}_{\mathrm{X}} \mathrm{CuO}_{4}$ at $x=x_{c}=0.185(5)$, and that this linear regime extends at fixed $x$ vertically all the way from $T=0$ right up to the highest $T$ studied $(200 \mathrm{~K})$. This result is inexplicable if one thinks of $(x, T)=\left(x_{c}, 0\right)$ as a "quantum critical point," but it is perfectly understandable in the context of a hard-wired ZZIP network, formed at the annealing temperature [30]. In fact, one can go further: the vertical linear $T$ regime is shaped like an hour glass, wide at $T=0$ and $T=200 \mathrm{~K}$, and narrow at $T \sim 100 \mathrm{~K}$. This narrowing is due to the formation of pseudogap islands, which requires a reformation of the nanodomain network at low $T$, into a doubly percolative network, with two kinds of dopants [30].

Naturally, percolative conductive paths are phasesensitive, and when this aspect is combined with the effects of long-range conductive screening of internal electric fields, it is easier to understand why descriptions of disorder using functions dependent on amplitudes alone (such as the participation ratio) are insufficient to describe filamentary self-organization [85]. Finally, it is worth mentioning that the philosophy employed here in the search for a satisfactory percolative solution to this exponentially complex glassy network problem [29] can be justified (after the fact of its success) not only heuristically but also by formal Boolean algebra [86]. This algebra shows that the determining factor in fixing the properties of exponentially complex systems is their topology, not their geometry [87, 88]. Of course, topology is a characteristic feature of networks, especially in strongly disordered layered structures, that is absent from continuum (ideal lattice) models.

\section{Acknowledgment}

I am grateful to Professor T. H. Geballe for stimulating my interest in MMU ceramic cuprate superconductors.

\section{References}

[1] K. A. Brueckner, T. Soda, P. W. Anderson, and P. Morel, "Level structure of nuclear matter and liquid $\mathrm{He}^{3}$," Physical Review, vol. 118, no. 5, pp. 1442-1446, 1960.

[2] R. C. Richardson, "The Pomeranchuk effect," Reviews of Modern Physics, vol. 69, no. 3, pp. 683-690, 1997.

[3] K. J. Chang, M. M. Dacorogna, M. L. Cohen, J. M. Mignot, G. Chouteau, and G. Martinez, "Superconductivity in highpressure metallic phases of Si," Physical Review Letters, vol. 54, no. 21, pp. 2375-2378, 1985.

[4] A. Y. Liu, I. I. Mazin, and J. Kortus, "Beyond Eliashberg superconductivity in $\mathrm{MgB}_{2}$ : anharmonicity, two-phonon scattering, and multiple gaps," Physical Review Letters, vol. 87, no. 8, Article ID 087005, 4 pages, 2001.

[5] J. E. Moussa and M. L. Cohen, "Constraints on Tc for superconductivity in heavily boron-doped diamond," Physical Review B, vol. 77, no. 6, Article ID 064518, 2008.

[6] C. Knight, S. J. Singer, J.-L. Kuo, T. K. Hirsch, L. Ojamäe, and M. L. Klein, "Hydrogen bond topology and the ice VII/VIII and Ih/XI proton ordering phase transitions," Physical Review E, vol. 73, no. 5, Article ID 056113, 2006.

[7] D. G. Schlom, L.-Q. Chen, C.-B. Eom, K. M. Rabe, S. K. Streiffer, and J.-M. Triscone, "Strain tuning of ferroelectric thin films," Annual Review of Materials Research, vol. 37, pp. 589-626, 2007.

[8] E. Şaşıŏ̆lu, L. M. Sandratskii, P. Bruno, and I. Galanakis, "Exchange interactions and temperature dependence of magnetization in half-metallic Heusler alloys," Physical Review B, vol. 72, Article ID 184415, 11 pages, 2005.

[9] J. C. Phillips, Physics of High- $T_{c}$ Superconductors , Academic Press, Boston, Mass, USA, 1989.

[10] P. W. Anderson, "The resonating valence bond state in $\mathrm{La}_{2} \mathrm{CuO}_{4}$ and superconductivity," Science, vol. 235, no. 4793, pp. 1196-1198, 1987.

[11] P. W. Anderson, P. A. Lee, M. Randeria, T. M. Rice, N. Trivedi, and F. C. Zhang, "The physics behind high-temperature superconducting cuprates: the 'plain vanilla' version of RVB," Journal of Physics: Condensed Matter, vol. 16, no. 24, pp. R755R769, 2004.

[12] A. Alexandrov and J. Ranninger, "Bipolaronic superconductivity," Physical Review B, vol. 24, no. 3, pp. 1164-1169, 1981.

[13] A. S. Alexandrov and N. F. Mott, "Bipolarons," Reports on Progress in Physics, vol. 57, no. 12, pp. 1197-1288, 1994.

[14] J. C. Phillips, "Giant defect-enhanced electron-phonon interactions in ternary copper oxide superconductors," Physical Review Letters, vol. 59, no. 16, pp. 1856-1859, 1987.

[15] F. Giustino, M. L. Cohen, and S. G. Louie, "Small phonon contribution to the photoemission kink in the copper oxide superconductors," Nature, vol. 452, no. 7190, pp. 975-978, 2008.

[16] J. C. Phillips and J. Jung, "Nanodomain structure and function of high-temperature superconductors," Philosophical Magazine B, vol. 81, no. 8, pp. 745-756, 2001.

[17] K. McElroy, J. Lee, J. A. Slezak, et al., "Atomic-scale sources and mechanism of nanoscale electronic disorder in $\mathrm{Bi}_{2} \mathrm{Sr}_{2} \mathrm{CaCu}_{2} \mathrm{O}_{8+\delta}$," Science, vol. 309, no. 5737, pp. 1048-1052, 2005.

[18] J. E. Moussa and M. L. Cohen, "Using molecular fragments to estimate electron-phonon coupling and possible superconductivity in covalent materials," Physical Review B, vol. 78, no. 6, Article ID 064502, 2008.

[19] K. M. Rabe, J. C. Phillips, P. Villars, and I. D. Brown, "Global multinary structural chemistry of stable quasicrystals, high- $T_{C}$ ferroelectrics, and high- $T_{c}$ superconductors," Physical Review $B$, vol. 45, no. 14, pp. 7650-7676, 1992.

[20] A. J. Leggett, "What do we know about high $T_{c}$ ?" Nature Physics, vol. 2, no. 3, pp. 134-136, 2006.

[21] H. Kamimura and M. Eto, " ${ }^{1} \mathrm{~A}_{1 \mathrm{~g}}$ to ${ }^{3} \mathrm{~B}_{1 \mathrm{lg}}$ conversion at the onset of superconductivity in $\mathrm{La}_{2-x} \mathrm{Sr}_{x} \mathrm{CuO}_{4}$ due to the apical oxygen effect," Journal of the Physical Society of Japan, vol. 59, no. 9, pp. 3053-3056, 1990.

[22] C. D. Castro, L. F. Feiner, and M. Grilli, "Symmetry of hole states in superconducting oxides: correlation with $T_{c}$," Physical Review Letters, vol. 66, no. 24, pp. 3209-3212, 1991.

[23] Y. Ohta, T. Tohyama, and S. Maekawa, "Apex oxygen and critical temperature in copper oxide superconductors: universal correlation with the stability of local singlets," Physical Review B, vol. 43, no. 4, pp. 2968-2982, 1991.

[24] J. C. Phillips, "Coherent resonant pinning, oxygen ordering, and high-temperature superconductivity in the multilayer cuprates," Physical Review Letters, vol. 72, no. 24, pp. 38633866, 1994. 
[25] H. Oesterreicher, "Plane isolation model for superconducting $T_{c}$ prediction using an 'parent-calibration' obtained for $\mathrm{Y}$ $\mathrm{Ba}_{2} \mathrm{CuCu}_{2} \mathrm{O}_{y}$ and applied to other model systems," Solid State Communications, vol. 142, no. 10, pp. 583-586, 2007.

[26] M. Mori, G. Khaliullin, T. Tohyama, and S. Maekawa, "Origin of the spatial variation of the pairing gap in Bi-based high temperature cuprate superconductors," Physical Review Letters, vol. 101, no. 24, Article ID 247003, 2008.

[27] X. Wang, H. Song, G. Qiu, and D. Wang, "Empirical criteria of superconductivity for some oxides," Journal of Materials Science and Technology, vol. 16, no. 3, pp. 327-331, 2000.

[28] J. C. Phillips, "Is there a lowest upper bound for superconductive transition temperatures?" Chemical Physics Letters, vol. 451, no. 1-3, pp. 98-101, 2008.

[29] P. Boolchand, G. Lucovsky, J. C. Phillips, and M. F. Thorpe, "Self-organization and the physics of glassy networks," Philosophical Magazine, vol. 85, no. 32, pp. 3823-3838, 2005.

[30] J. C. Phillips, "Self-organized networks and lattice effects in high-temperature superconductors," Physical Review B, vol. 75, no. 21, Article ID 214503, 2007.

[31] H. Uchiyama, A. Q. R. Baron, S. Tsutsui, et al., "Softening of $\mathrm{Cu}-\mathrm{O}$ bond stretching phonons in tetragonal $\mathrm{HgBa}_{2} \mathrm{CuO} \mathrm{O}_{4+\delta}$," Physical Review Letters, vol. 92, no. 19, Article ID 197005, 4 pages, 2004.

[32] S. L. Chaplot, W. Reichardt, L. Pintschovius, and N. Pyka, "Common interatomic potential model for the lattice dynamics of several cuprates," Physical Review B, vol. 52, no. 10, pp. 7230-7242, 1995.

[33] A. D. Christianson, M. D. Lumsden, O. Delaire, et al., "Phonon density of states of $\mathrm{LaFeAsO}_{1-x} \mathrm{~F}_{x}$ " Physical Review Letters, vol. 101, no. 15, Article ID 157004, 2008.

[34] S. Shimizu, H. Mukuda, Y. Kitaoka, et al., "Self-doped superconductivity in tri-layered $\mathrm{Ba}_{2} \mathrm{Ca}_{2} \mathrm{Cu}_{3} \mathrm{O}_{6} \mathrm{~F}_{2}$ : a ${ }^{63} \mathrm{Cu}$ NMR study," Physica B, vol. 403, no. 5-9, pp. 1041-1043, 2008.

[35] J. C. Phillips, "Direct evidence for the quantum interlayer defect-assisted percolation model of cuprate high- $T_{c}$ superconductivity," Physical Review B, vol. 39, no. 10, pp. 73567358, 1989.

[36] S. Yamanaka, K.-I. Hotehama, and H. Kawaji, "Superconductivity at $25.5 \mathrm{~K}$ in electron-doped layered hafnium nitride," Nature, vol. 392, no. 6676, pp. 580-582, 1998.

[37] Y. Kamihara, T. Watanabe, M. Hirano, and H. Hosono, "Ironbased layered superconductor $\mathrm{La}\left[\mathrm{O}_{1-x} \mathrm{~F}_{x}\right]$ FeAs $(x=0.05-$ 0.12) with $T_{c}=26 \mathrm{~K}$," Journal of the American Chemical Society, vol. 130, no. 11, pp. 3296-3297, 2008.

[38] J. C. Phillips, "Nanostructural model of metal-insulator transition in layered Lix ZrNCl superconductors," Physical Review B, vol. 77, no. 10, Article ID 104534, 2008.

[39] D. J. Singh and M.-H. Du, "Density functional study of $\mathrm{LaFeAsO}_{1-x} \mathrm{~F}_{x}$ : a low carrier density superconductor near itinerant magnetism," Physical Review Letters, vol. 100, no. 23, Article ID 237003, 2008.

[40] G. Saffarini, J. M. Saiter, and J. Matthiesen, “Thermal stability and percolation threshold of Ge-Se-Fe glasses," Materials Letters, vol. 61, no. 2, pp. 432-436, 2007.

[41] D. J. Singh, "Electronic structure and doping in $\mathrm{BaFe}_{2} \mathrm{As}_{2}$ and LiFeAs: density functional calculations," Physical Review B, vol. 78, no. 9, Article ID 094511, 2008.

[42] H. Takahashi, K. Igawa, K. Arii, Y. Kamihara, M. Hirano, and $\mathrm{H}$. Hosono, "Superconductivity at $43 \mathrm{~K}$ in an iron-based layered compound $\mathrm{LaO}_{1-x} \mathrm{~F}_{x} \mathrm{FeAs}$," Nature, vol. 453, no. 7193, pp. 376-378, 2008.
[43] M. S. Torikachvili, S. L. Bud'ko, N. Ni, and P. C. Canfield, "Effect of pressure on the structural phase transition and superconductivity in $\left(\mathrm{Ba}_{1-x} \mathrm{~K}_{x}\right) \mathrm{Fe}_{2} \mathrm{As}_{2}(x=0$ and 0.45$)$ and $\mathrm{SrFe}_{2} \mathrm{As}_{2}$ single crystals," Physical Review B, vol. 78, no. 10, Article ID 104527, 6 pages, 2008.

[44] A. Gozar, G. Logvenov, L. F. Kourkoutis, et al., "Hightemperature interface superconductivity between metallic and insulating copper oxides," Nature, vol. 455, no. 7214, pp. 782785,2008 .

[45] S. Reich and Y. Tsabba, "Possible nucleation of a 2D superconducting phase on $\mathrm{WO}_{3}$ single crystals surface doped with $\mathrm{Na}^{+}$," European Physical Journal B, vol. 9, no. 1, pp. 1-4, 1999.

[46] G. Leitus, H. Cohen, and S. Reich, "Interplay of Cs concentration, dimensionality and superconductivity in $\mathrm{Cs}_{x} \mathrm{WO}_{3}$," Physica C, vol. 371, no. 4, pp. 321-329, 2002.

[47] A. E. Aliev, "High- $T_{c}$ superconductivity in nanostructured $\mathrm{Na}_{\mathrm{x}} \mathrm{WO}_{3-y}$ : sol-gel route," Superconductor Science and Technology, vol. 21, no. 11, Article ID 115022, 9 pages, 2008.

[48] Y. Kopelevich, R. R. da Silva, A. Rougier, and I. A. Luk'yanchuk, "Charge ordering in amorphous $\mathrm{WO}_{x}$ films," Physics Letters, vol. 368, no. 5, pp. 419-422, 2007.

[49] T. Takano, T. Kishiume, Y. Taguchi, and Y. Iwasa, "Interlayerspacing dependence of $T_{c}$ in $\mathrm{Li}_{x} M_{y} \mathrm{HfNCl}$ ( $M$ : molecule) superconductors," Physical Review Letters, vol. 100, no. 24, Article ID 247005, 4 pages, 2008.

[50] F. Carbone, D.-S. Yang, E. Giannini, and A. H. Zewail, "Direct role of structural dynamics in electron-lattice coupling of superconducting cuprates," Proceedings of the National Academy of Sciences of the United States of America, vol. 105, no. 51, pp. 20161-20166, 2008.

[51] T. P. Devereaux, T. Cuk, Z.-X. Shen, and N. Nagaosa, "Anisotropic electron-phonon interaction in the cuprates," Physical Review Letters, vol. 93, no. 11, Article ID 117004, 4 pages, 2004.

[52] V. A. Rassolov and S. Garashchuk, "Computational complexity in quantum chemistry," Chemical Physics Letters, vol. 464, no. 4-6, pp. 262-264, 2008.

[53] H. Shim, P. Chaudhari, G. Logvenov, and I. Bozovic, "Electron-phonon interactions in superconducting $\mathrm{La}_{1.84} \mathrm{Sr}_{0.16} \mathrm{CuO}_{4}$ films," Physical Review Letters, vol. 101, no. 24, Article ID 247004, 4 pages, 2008.

[54] J. C. Phillips, "Electron-phonon interactions cause hightemperature superconductivity," Philosophical Magazine B, vol. 82, no. 8, pp. 931-942, 2002.

[55] H. Iwasawa, J. F. Douglas, K. Sato, et al., "Isotopic fingerprint of electron-phonon coupling in high- $T_{c}$ cuprates," Physical Review Letters, vol. 101, no. 15, Article ID 157005, 2008.

[56] D. Lampakis, E. Liarokapis, and C. Panagopoulos, "MicroRaman evidence for topological charge order across the superconducting dome of $\mathrm{La}_{2-x} \mathrm{Sr}_{x} \mathrm{CuO}_{4}$," Physical Review $B$, vol. 73, no. 17, Article ID 174518, 2006.

[57] A. Bianconi, M. Lusignoli, N. L. Saini, P. Bordet, Å. Kvick, and P. G. Radaelli, "Stripe structure of the $\mathrm{CuO}_{2}$ plane in $\mathrm{Bi}_{2} \mathrm{Sr}_{2} \mathrm{CaCu}_{2} \mathrm{O}_{8+y}$ by anomalous X-ray diffraction," Physical Review B, vol. 54, no. 6, pp. 4310-4314, 1996.

[58] N. Barišić, Y. Li, X. Zhao, et al., "Demonstrating the model nature of the high-temperature superconductor $\mathrm{HgBa}_{2} \mathrm{CuO}_{4+\delta}$," Physical Review B, vol. 78, no. 5, Article ID 054518, 2008.

[59] C. J. Zhang and H. Oyanagi, "Local lattice instability and superconductivity in $\mathrm{La}_{1.85} \mathrm{Sr}_{0.15} \mathrm{Cu}_{1-x} M_{\mathrm{x}} \mathrm{O}_{4}(M=\mathrm{Mn}, \mathrm{Ni}$, and Co)," Physical Review B, vol. 79, no. 6, Article ID 064521, 8 pages, 2009. 
[60] F. Illas, D. Muñoz, C. de Graaf, and I. de P. R. Moreira, "Unexpected role of Madelung potential in monoplanar high$T_{c}$ cuprate superconductors," Chemical Physics Letters, vol. 379, no. 3-4, pp. 291-296, 2003.

[61] M. C. Boyer, W. D. Wise, K. Chatterjee, et al., "Imaging the two gaps of the high-temperature superconductor $\mathrm{Bi}_{2} \mathrm{Sr}_{2} \mathrm{CuO}_{6+x}$," Nature Physics, vol. 3, no. 11, pp. 802-806, 2007.

[62] J.-H. Ma, Z.-H. Pan, F. C. Niestemski, et al., "Coexistence of competing orders with two energy gaps in real and momentum space in the high temperature superconductor $\mathrm{Bi}_{2} \mathrm{Sr}_{2-x} \mathrm{La}_{x} \mathrm{CuO}_{6+\delta}$," Physical Review Letters, vol. 101, no. 20, Article ID 207002, 2008.

[63] G. Leibon, S. Pauls, D. Rockmore, and R. Savell, "Topological structures in the equities market network," Proceedings of the National Academy of Sciences of the United States of America, vol. 105, no. 52, pp. 20589-20594, 2008.

[64] S. Saavedra, F. Reed-Tsochas, and B. Uzzi, "Asymmetric disassembly and robustness in declining networks," Proceedings of the National Academy of Sciences of the United States of America, vol. 105, no. 43, pp. 16466-16471, 2008.

[65] K. Venkatesan, J.-F. Rual, A. Vazquez, et al., "An empirical framework for binary interactome mapping," Nature Methods, vol. 6, no. 1, pp. 83-90, 2009.

[66] M. A. Moret and G. F. Zebende, "Amino acid hydrophobicity and accessible surface area," Physical Review E, vol. 75, no. 1, Article ID 011920, 2007.

[67] A. E. Kister and J. C. Phillips, "A stringent test for hydrophobicity scales: two proteins with $88 \%$ sequence identity but different structure and function," Proceedings of the National Academy of Sciences of the United States of America, vol. 105, no. 27, pp. 9233-9237, 2008.

[68] J. C. Phillips, "Quantum percolation in cuprate hightemperature superconductors," Proceedings of the National Academy of Sciences of the United States of America, vol. 105, no. 29, pp. 9917-9919, 2008.

[69] J. Yang, J. Hwang, E. Schachinger, et al., "Exchange boson dynamics in cuprates: optical conductivity of $\mathrm{HgBa}_{2} \mathrm{CuO}_{4+\delta}$," Physical Review Letters, vol. 102, no. 2, Article ID 027003, 2009.

[70] W. Guyard, A. Sacuto, M. Cazayous, et al., "Temperature dependence of the gap size near the brillouin-zone nodes of $\mathrm{HgBa}_{2} \mathrm{CuO}_{4+\delta}$ superconductors," Physical Review Letters, vol. 101, no. 9, Article ID 097003, 2008.

[71] S. Cojocaru, R. Citro, and M. Marinaro, "Incoherent midinfrared charge excitation and the high-energy anomaly in the photoemission spectra of cuprates," Physical Review B, vol. 75, no. 22, Article ID 220502, 2007.

[72] C. U. Jung, J. H. Kong, B. H. Park, T. W. Noh, and E. J. Choi, "Far-infrared transmission studies on a superconducting $\mathrm{BaPb}_{1-x} \mathrm{Bi}_{x} \mathrm{O}_{3}$ thin film: effects of a carrier scattering rate," Physical Review B, vol. 59, no. 13, pp. 8869-8874, 1999.

[73] T. Nishio, J. Ahmad, and H. Uwe, "Spectroscopic observation of bipolaronic point defects in $\mathrm{Ba}_{1-x} \mathrm{~K}_{x} \mathrm{BiO}_{3}$," Physical Review Letters, vol. 95, no. 17, Article ID 176403, 4 pages, 2005.

[74] K. Haule, J. H. Shim, and G. Kotliar, "Correlated electronic structure of $\mathrm{LaO}_{1-x} \mathrm{~F}_{x} \mathrm{FeAs}$," Physical Review Letters, vol. 100, no. 22, Article ID 226402, 2008.

[75] T. Yildirim, "Strong coupling of the Fe-Spin state and the AsAs hybridization in Iron-Pnictide superconductors from firstprinciple calculations," Physical Review Letters, vol. 102, no. 3, Article ID 037003, 2009.

[76] I. Nowik and I. Felner, "Mössbauer spectroscopy determination of iron foreign phases in the superconducting systems; RAsFeO ${ }_{1-x}, R \mathrm{AsFeO}_{1-x} \mathrm{~F}_{x}$, and $\mathrm{Sr}_{1-x} \mathrm{~K}_{x} \mathrm{Fe}_{2} \mathrm{As}_{2}$," Journal of
Superconductivity and Novel Magnetism, vol. 21, no. 5, pp. 297-300, 2008.

[77] A. S. Alexandrov, "Phase separation of electrons strongly coupled with phonons in cuprates and manganites," Journal of Superconductivity and Novel Magnetism, vol. 22, no. 2, pp. 95-101, 2009.

[78] A. V. Boris, N. N. Kovaleva, S. S. A. Seo, et al., "Signatures of electronic correlations in optical properties of $\mathrm{LaFeAsO}_{1-x} \mathrm{~F}_{x}$," Physical Review Letters, vol. 102, no. 2, Article ID 027001, 2009.

[79] A. S. Alexandrov, "Unconventional pairing symmetry of layered superconductors caused by acoustic phonons," Physical Review B, vol. 77, no. 9, Article ID 094502, 2008.

[80] H. Q. Yuan, J. Singleton, F. F. Balakirev, et al., "Nearly isotropic superconductivity in $(\mathrm{Ba}, \mathrm{K}) \mathrm{Fe}_{2} \mathrm{As}_{2}$," Nature, vol. 457, no. 7229, pp. 565-568, 2009.

[81] T. Ekino, A. M. Gabovich, M. S. Li, M. Pekała, H. Szymczak, and A. I. Voitenko, "Temperature-dependent pseudogaplike features in tunnel spectra of high- $T_{c}$ cuprates as a manifestation of charge-density waves," Journal of Physics: Condensed Matter, vol. 20, no. 42, Article ID 425218, 2008.

[82] Y. Ando, S. Komiya, K. Segawa, S. Ono, and Y. Kurita, "Electronic phase diagram of high- $T_{c}$, cuprate superconductors from a mapping of the In-plane resistivity curvature," Physical Review Letters, vol. 93, no. 26, Article ID 267001, 2004.

[83] J. Jung and M. M. Abdelhadi, "Electrical transport and oxygen disorder in YBCO," International Journal of Modern Physics B, vol. 17, no. 18-20, pp. 3465-3469, 2003.

[84] R. A. Cooper, Y. Wang, B. Vignolle, et al., "Anomalous criticality in the electrical resistivity of $\mathrm{La}_{2-x} \mathrm{Sr}_{x} \mathrm{CuO}_{4}$," Science, vol. 323, no. 5914, pp. 603-607, 2009.

[85] M. F. Islam and H. Nakanishi, "The effect of local phases of wave function on transmission of a quantum particle through two dimensional clusters," European Physical Journal B, vol. 65, no. 4, pp. 555-564, 2008.

[86] S. Cocco and R. Monasson, "Exponentially hard problems are sometimes polynomial, a large deviation analysis of search algorithms for the random satisfiability problem, and its application to stop-and-restart resolutions," Physical Review E, vol. 66, no. 3, Article ID 037101, 4 pages, 2002.

[87] B. Luque, O. Miramontes, and L. Lacasa, "Number theoretic example of scale-free topology inducing self-organized criticality," Physical Review Letters, vol. 101, no. 15, Article ID 158702, 2008.

[88] T. Rohlf, "Self-organization of heterogeneous topology and symmetry breaking in networks with adaptive thresholds and rewiring," Europhysics Letters, vol. 84, no. 1, Article ID 10004, 2008. 

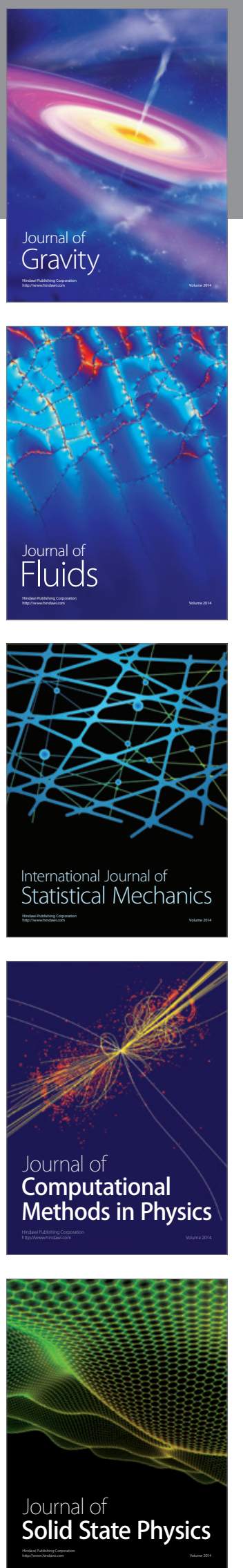

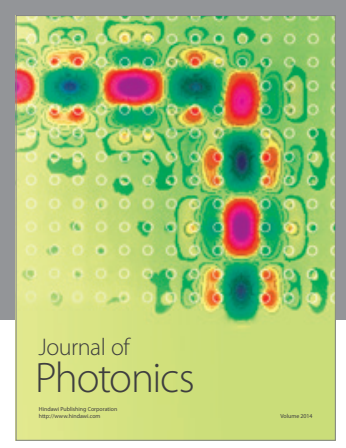

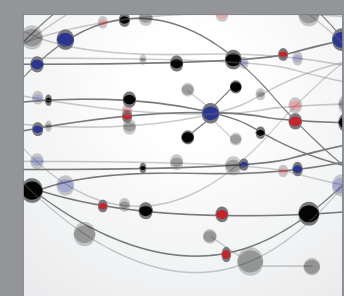

The Scientific World Journal
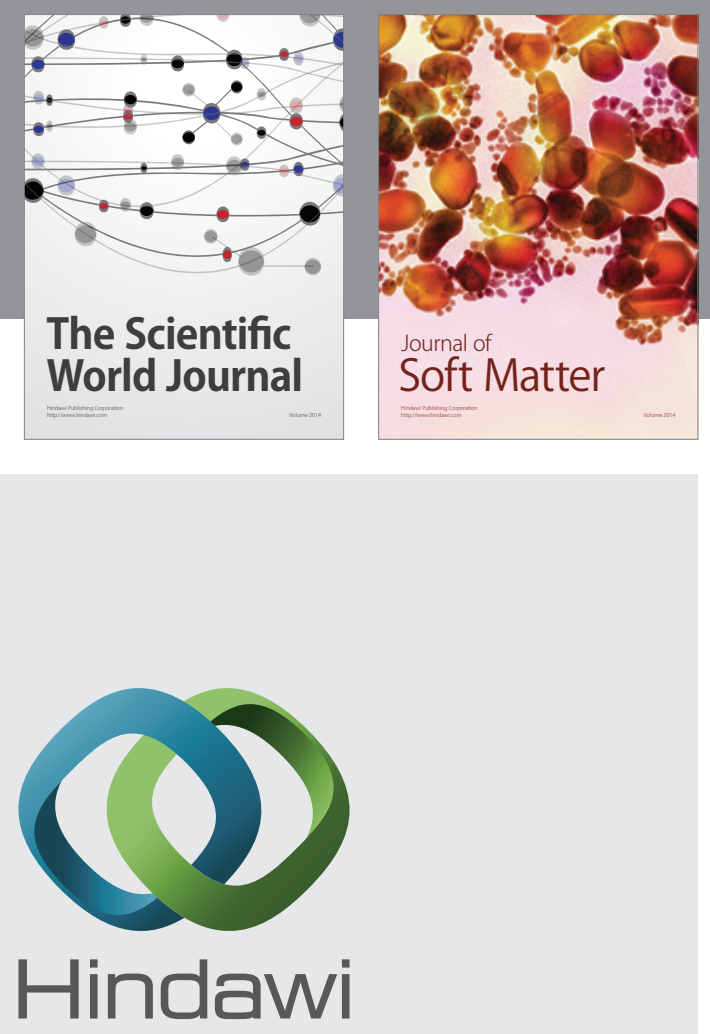

Submit your manuscripts at

http://www.hindawi.com
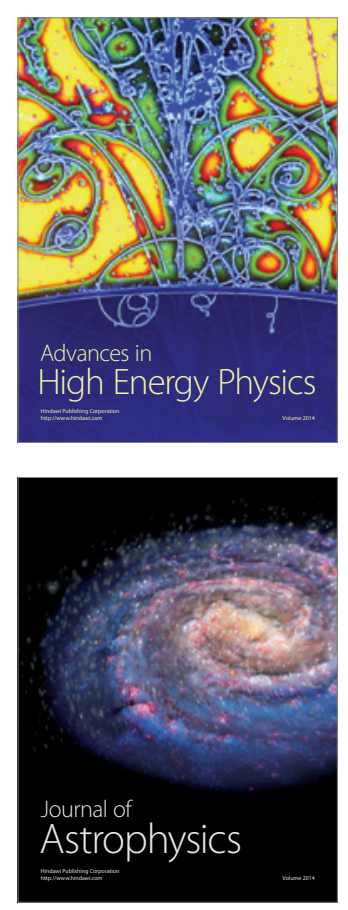
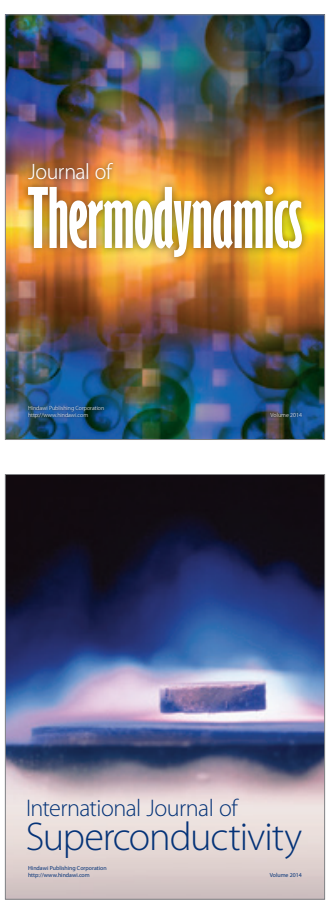
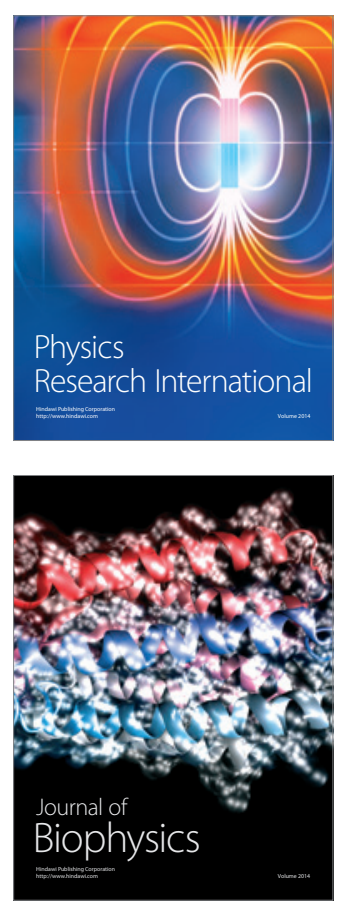
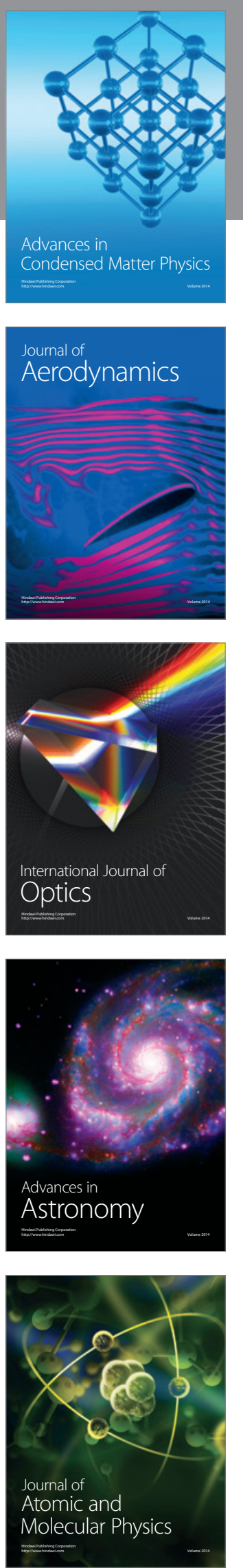